\title{
Effects and Interaction of Icariin, Curculigoside, and Berberine in Er-Xian Decoction, a Traditional Chinese Medicinal Formula, on Osteoclastic Bone Resorption
}

\author{
Liming Xue, ${ }^{1}$ Lei Jiao, ${ }^{1}$ Yin Wang, ${ }^{2}$ Yan Nie, ${ }^{1}$ Ting Han, ${ }^{1}$ \\ Yiping Jiang, ${ }^{1}$ Khalid Rahman, ${ }^{3}$ Qiaoyan Zhang, ${ }^{1}$ and Luping Qin ${ }^{1}$ \\ ${ }^{1}$ School of Pharmacy, Second Military Medical University, 325 Guohe Road, Shanghai 200433, China \\ ${ }^{2}$ Department of Pharmaceutical, PLA 455 Hospital, Shanghai 200052, China \\ ${ }^{3}$ School of Pharmacy and Biomolecular Sciences, Liverpool John Moores University, Byrom Street, Liverpool L3 3AF, UK
}

Correspondence should be addressed to Qiaoyan Zhang, zqy1965@163.com and Luping Qin, lpqin@smmu.edu.cn

Received 11 July 2012; Revised 9 September 2012; Accepted 24 September 2012

Academic Editor: Norazlina Mohamed

Copyright ( $) 2012$ Liming Xue et al. This is an open access article distributed under the Creative Commons Attribution License, which permits unrestricted use, distribution, and reproduction in any medium, provided the original work is properly cited.

\begin{abstract}
Er-Xian decoction (EXD), a traditional Chinese medicine, has been reported to have a protective effect against bone loss in ovariectomized osteoporotic rats, and the inclusion of icariin (I), curculigoside (C), and berberine (B) in EXD displays inhibitory effects on osteoclastic bone resorption. In the present paper, we investigated the interaction and effects of I, C, B, and their combination on bone resorption activity in vitro on osteoclasts derived from rat bone marrow cells. ICB synergistically decreased the formation of bone resorption pits, the number of multinucleated osteoclasts, and the activity of tartrate-resistant acid phosphatase (TRAP) and showed antagonistic or additive effects on cathepsin K activity in the coculture system of osteoblasts and bone marrow cells in the presence of 1,25-dihydroxyvitamin $\mathrm{D}_{3}$ and dexamethasone. The combination of ICB also enhanced the inhibitory effects on the formation of F-actin ring, a cytoskeleton structure of osteoclasts induced from bone marrow cells with macrophage colony stimulation factor (M-CSF) and receptor activator of NF- $\kappa$ B ligand (RANKL). In addition, ICB synergistically improved the ratio of protein expression of osteoprotegerin (OPG) and RANKL in osteoblasts and interfered with the mitogenactivated protein kinases (MAPKs) pathway in osteoclast. These results clearly show that I, C, B, and their combination in EXD exert effects of mutual reinforcement. However, IBC does not show an intensified adverse effect in the ovariectomized murine model, as revealed by change in body and uterine weight, confirming the safety of EXD. These observations are in agreement with the rationality of the formula used in this paper.
\end{abstract}

\section{Introduction}

Traditional Chinese medicine (TCM), an empirical system of multicomponent therapeutics, potentially meets the demands of treating a number of complex diseases in an integrated manner, in particular chronic diseases and metabolic syndromes [1]. Naturally occurring herbs and herbal ingredients organized into certain formula have been shown to have potential interaction effects, including mutual enhancement, mutual assistance, mutual restraint, and mutual antagonism. For example, synergistic interactions occur when the efficacy of combinations of herbs (or ingredients) is greater than the summed responses of each individual herb or ingredient $[2,3]$. Scientific evidence for TCM is generally achieved through rigorous experimental design, which has been dominated by the search for its biological basis, identification of active substances, and the investigation of the mechanisms of its action. In contrast, research in the role and interaction of active ingredients in the formulas is still scarce, thus hampering the understanding of the rationality of formula design in TCM.

Postmenopausal osteoporosis is believed to be associated with ovarian hormone deficiency and is by far the most common cause of age-related bone loss [4]; and with the reduction in estrogen levels, there is an increase in bone breakdown relative to bone formation, microarchitectural 
deterioration, and decreased bone mass $[4,5]$. The excessive bone breakdown is caused by an increased number of osteoclast and enhanced ability of bone resorption [6]. Osteoclasts are multinucleated cells (3-10 nuclei per cell) differentiated from hematopoietic precursors and are involved in bone remodeling. Through osteoclastic bone resorption, osteoclasts play both a crucial physiological role in bone remodeling and a pathological role in osteoporosis as characterized by excess activity of osteoclasts $[6,7]$.

The osteoclasts are formed and differentiated under the control of some cytokines, such as macrophage colony stimulation factor (M-CSF), receptor activator of NF$\kappa \mathrm{B}$ ligand (RANKL), and osteoprotegerin (OPG) $[7,8]$. The binding of RANKL to its receptor RANK leads to recruitment of TNF receptor-associated factor 6 (TRAF6) to the cytoplasmic domain of RANK. This then activates the downstream targets of TRAF6 including transcription factors such as nuclear factor kappa B (NF- $\kappa \mathrm{B})$, activator protein-1 (AP-1), and nuclear factor of activated $\mathrm{T}$ cells (NFAT), as well as the mitogen-activated protein kinases (MAPKs) including p38 MAP kinases, c-Jun $\mathrm{N}$-terminal kinases (JNK/SAPKs), extracellular signal-regulated kinases (ERKs), and phosphatidyl-inositol-3-kinase (PI3 K)/Akt [912]. Osteoprotegerin (OPG), a soluble decoy receptor of RANKL, negatively regulates osteoclast differentiation and bone resorption. Mature osteoclast formation is also associated with the expression of differentiation markers, including tartrate resistant acid phosphatase (TRAP) and cathepsin $\mathrm{K}$. The modulation of these differentiation-related signaling pathways has the potential of being considered as a therapeutic strategy for the treatment of skeletal diseases [13].

Over the past 50 years, Er-Xian decoction (EXD), a traditional Chinese medicinal formula, has been used for the treatment of osteoporosis disorders, menopausal syndrome, and aging diseases [14, 15]. The components of EXD are Epimedii Folium (Yinyanghuo), Curculiginis Rhizoma (Xianmao), Anemarrhenae Rhizoma (Zhimu), Phellodendri Chinensis Cortex (Huangbo), Morindae Officinalis Radix (Bajitian), and Angelicae Sinensis Radix (Danggui), with icariin, curculigoside, timosaponin BII, berberin, nystose, and ferulic acid as major active ingredients [16]. According to traditional Chinese medicine, Epimedium Folium and Phellodendri Chinensis Cortex are essential ingredients of EXD and appear to play important roles in ameliorating signs and symptoms of the menopausal syndrome and osteoporosis. Curculiginis Rhizoma and Morindae Officinalis Radix help strengthen the curative effect of the Epimedium Folium, and Anemarrhenae Rhizoma helps Phellodendri Chinensis Cortex to play a fuller role. Angelicae Sinensis Radix cooperates with the above five medications to strengthen the therapeutic effects and treat the accompanying disease or syndromes [17]. We tested the effects of major active ingredients on osteoblast and osteoclast and found that icariin, timosaponin BII, nystose, and ferulic acid could increase the proliferation and alkaline phosphatase (ALP) activity of osteoblast, and icariin (I), curculigoside (C), and berberine (B) could decrease the number of TRAP positive multinucleated osteoclasts and TRAP-activity of osteoclast, as evidenced by their inhibitory effects on bone resorption [16].

Therefore, in the present study, we firstly proved that the combination of ICB has more potent antiosteoporotic activity than individual compounds on ovariectomized osteoporotic mice, and furthermore, we investigated the inhibitory effects of I, C, B, and their combination on bone resorption of octeoclast to evaluate the interaction between them so as to obtain an understanding of their mechanism of action and to provide an insight into the rationality of formula design for potential use in TCM.

\section{Materials and Methods}

2.1. Reagents. Reagents used in this study included $\alpha$ modified minimum essential medium $(\alpha$-MEM) and fetal bovine serum (FBS) (Gibco, US); 1, 25-dihydroxyvitamin $\mathrm{D}_{3}$, dexamethasone, trypsin, and coomassie brilliant blue G-250 (Sigma, US); recombinant rat M-CSF (400-28) and RANKL (400-30) (Peprotech EC, US); OPG (sc11383), RANKL (sc9073), and $\beta$-actin (sc81178) antibody (Santa Cruz, USA); anti-phospho-Akt (9275), anti-phospho-ERK (3371), anti-phospho-JNK (9251), anti-phospho-p38 (9211), and anti-phospho-I- $\kappa$ B (9245) (Cell Signaling Technology, Beverly, MA); NucBuster Protein Extraction kit (Merck, Novagen, Germany); Cathepsin K activity kit (Biovision, USA); pararosaniline, Hoechst 33258, and rhodamine-conjugated phalloidin (Sigma, USA). Diolamine, potassium sodium tartrate, disodium 4nitrophenylphosphate, Triton X-100, and 4-nitrophenol were of domestic AR grade.

Icariin (I), curculigoside (C), and berberine (B) were isolated from Epimedii Folium, Curculiginis Rhizoma, and Phellodendri Chinensis Cortex, respectively, and were identified by spectrum analysis. The data of proton nuclear magnetic resonance spectroscopy ( ${ }^{1} \mathrm{H}$ NMR $)$ and mass spectrometry (MS) were coincidence with previous studies. The purities of icariin, curculigoside, and berberine were up to 99\% according to high-performance liquid chromatography (HPLC) analysis.

\subsection{Animal Experimental Protocol. Seventy 3-month-old} female ICR mice were purchased from SLACOM experimental animal company (Shanghai, China) and acclimatized to laboratory conditions for 1 week before commencing the experiment. Food and drinking water were supplied ad libitum. The mice were weighed weekly during the experimental period. An osteoporotic model was established 12 weeks after bilateral ovariectomy. Knowing that the osteoporotic model induced by ovariectomy is associated with estrogen deficiency, nylestriol (an estrogenic substance) was used as the positive drug. Of the 70 female mice, 10 mice were sham-operated and treated with deionized water as the control group. The remaining 60 mice were bilaterally ovariectomized and equally randomized into six groups, with intragastric administration of either deionized water as the OVX model control, nylestriol $(1 \mathrm{mg} / \mathrm{kg})$ weekly as the positive control, icariin $(40 \mathrm{mg} / \mathrm{kg})$ daily, curculigoside 
$(20 \mathrm{mg} / \mathrm{kg})$ daily, berberine $(120 \mathrm{mg} / \mathrm{kg})$ daily, or a combination of icariin $(40 \mathrm{mg} / \mathrm{kg})$, curculigoside $(20 \mathrm{mg} / \mathrm{kg})$, and berberine $(120 \mathrm{mg} / \mathrm{kg})$ daily as the treatment groups, all for 12 weeks. Mice received treatments starting from day one after surgeries and the success of ovariectomy was confirmed at necropsy by failure to detect ovarian tissue and by observation of marked atrophy of uterine horns. At the end of the treatment, blood samples from all the mice were withdrawn by orbital sinus method and centrifuged to collect serum for the measurement of biochemical parameters. The uterine was removed and immediately weighed.

The tibia was cleaned by removing adhering soft tissues and stored in $75 \%$ ethanol for a week prior to analysis. The bone mineral density (BMD) and bone mineral content (BMC) were measured at $3 \mathrm{~mm}$ from the proximal epiphysis of right tibia with a peripheral quantitative computed tomography ( $p \mathrm{QCT}$ ) densitometry (Stratec XCT Research SA, Germany). Serum alkaline phosphatase (ALP), tartrateresistant acid phosphatase (TRAP), and serum creatinine (Cr) were measured on an automatic analyzer (Ciba-Corning 550, USA) using diagnostic reagent kit determination. Serum osteoprotegerin (OPG) and deoxypyridinoline cross-links (DPD) were estimated using an Elisa kit according to manufacturer instructions. The experimental protocol used in this study was approved by the Bioethic Committee of the Second Military Medical University, and the procedures employed were strictly according to generally accepted international rules and regulations.

\subsection{The Assay of Formation, Differentiation, and Bone Resorption of Osteoclast}

2.3.1. Osteoclasts Induced with 1, 25-Dihydroxyvitamin $D_{3}$ and Dexamethasone from Bone Marrow Cells in a Coculture System with Osteoblasts. Primary osteoblastic cells were prepared from neonatal rat calvarial osteoblasts according to the literature [18]. Induction and culture of osteoclasts were as follows. Briefly, the femur was disarticulated from Wistar rats aged 3 days, and the ends were removed, and the bone marrow cells were flushed out using a $1 \mathrm{~mL}$ syringe. Primary osteoblastic cells $\left(1 \times 10^{5} / \mathrm{mL}\right)$ and bone marrow cells $\left(1 \times 10^{6} / \mathrm{mL}\right)$ were cocultured in $\alpha$-MEM medium containing 10\% FBS, 1, 25-dihydroxyvitamin $\mathrm{D}_{3}$ $(10 \mathrm{nmol} / \mathrm{L})$ and dexamethasone $(100 \mathrm{nmol} / \mathrm{L})$ at $37^{\circ} \mathrm{C}$ in a humidified atmosphere of $5 \% \mathrm{CO}_{2}$. Prior to plating the cells, cover glasses $(5 \times 5 \mathrm{~mm})$ or dental slices $(40 \mu \mathrm{m}$ thick) were placed into culture dishes. The formations of multinucleated osteoclasts (MNCs) were confirmed by TRAP staining and resorption pits formed on dental slices. Osteoclastic cells were seeded onto culture plates in $\alpha$-MEM medium. The medium was replaced the following day with fresh medium containing $1.0 \mu \mathrm{M}$ icariin, $1.0 \mu \mathrm{M}$ curculigoside, $1.0 \mu \mathrm{M}$ berberine, or their combination (including $1.0 \mu \mathrm{M}$ icariin, $1.0 \mu \mathrm{M}$ curculigoside, and $1.0 \mu \mathrm{M}$ berberine) for indicated time. All cultures were maintained at $37^{\circ} \mathrm{C}$ in a humidified atmosphere of $5 \% \mathrm{CO}_{2}$ throughout. The osteoclasts were used to investigate formation of osteoclast, TRAP and Cathepsin $\mathrm{K}$ activity, and bone resorption activity.

\subsubsection{Counting of TRAP-Positive Multinucleated Osteoclasts.} Primary osteoblasts and bone marrow cells were placed on 96-well plates containing cover glasses and cultured in $\alpha$ MEM medium containing 10\% FBS, 1, 25-dihydroxyvitamin $\mathrm{D}_{3}(10 \mathrm{nM})$ and dexamethasone $(100 \mathrm{nM})$, for $24 \mathrm{~h}$. Cells were then treated with or without I, C, B, and their combination for 10 days and stained for TRAP with a TRAP staining kit (no. 387A-1KT; Sigma-Aldrich). Cells with three or more nuclei were counted as osteoclast-like multinucleated osteoclasts under a microscope.

2.3.3. Assay for TRAP Activity. Primary osteoblasts and bone marrow cells in $\alpha$-MEM medium containing 10\% FBS, 1, 25-dihydroxyvitamin $\mathrm{D}_{3}(10 \mathrm{nM})$, and dexamethasone $(100 \mathrm{nM})$ were placed in a 96-well culture dishes, cultured for 6 days, and then treated with or without I, C, B, and their combination for $48 \mathrm{~h}$. TRAP was determined as follows: cells were washed twice with PBS, and then $20 \mu \mathrm{L} 0.1 \%$ Triton X-100 was added to the cells to induce lysis at room temperature. After $15 \mathrm{~min}, 100 \mu \mathrm{L}$ substrate solution ( $0.4 \mathrm{~g}$ disodium 4 -nitrophenylphosphate and $2.0 \mathrm{~g}$ potassium tartrate dissolved in $200 \mathrm{~mL}$ of deionized water, with a $\mathrm{pH}$ adjusted to 3.5 with $1 \mathrm{~mol} / \mathrm{L} \mathrm{HCl}$ ) was added to the lysed cells and incubated at $37^{\circ} \mathrm{C}$ for $30 \mathrm{~min}$, the reaction was terminated by the addition of $100 \mu \mathrm{L} 1 \mathrm{~mol} / \mathrm{L} \mathrm{NaOH}$ to each well, and the absorbance was measured at $405 \mathrm{~nm}$. At the same time, positive cells for TRAP were counted and the TRAP activity was expressed as nanomoles $p$-nitrophenol per minute per 100 osteoclasts.

2.3.4. Determination of Cathepsin K Activity. Primary osteoblasts $\left(2 \times 10^{6}\right)$ and bone marrow cells $\left(2 \times 10^{7}\right)$ in $\alpha$-MEM medium containing $10 \%$ FBS, 1,25 -dihydroxyvitamin $\mathrm{D}_{3}$ $(10 \mathrm{nM})$, and dexamethasone $(100 \mathrm{nM})$ were placed in a 24 well culture plate, cultured for 7 days, and then treated with or without I, C, B, and their combination for $48 \mathrm{~h}$. Cells were collected by centrifugation, lysed in $50 \mu \mathrm{L}$ chilled CK cell lysis buffer, incubated on ice for $10 \mathrm{~min}$, and then vortexed for $5 \mathrm{~min}$. CK reaction buffer $(50 \mu \mathrm{L})$ and $10 \mathrm{mM} \mathrm{CK}$ substrate Ac-LR-AFC $(2 \mu \mathrm{L})$ were then added to the sample and the mixture solution was incubated at $37^{\circ} \mathrm{C}$ for $2 \mathrm{~h}$. The samples were then transferred onto a 96-well plate; the intensity of fluoresce was measured in a fluorometer equipped with a $400 \mathrm{~nm}$ excitation filter and a $505 \mathrm{~nm}$ emission filter.

2.3.5. Determination of Bone Resorption Pit. The primary osteoblast and bone marrow cell suspension were seeded into the wells of 96-well culture plates with a sterilized bone slice ( $40 \mu \mathrm{m}$ thick, $5 \mathrm{~mm} \times 5 \mathrm{~mm}$ ). After $24 \mathrm{~h}$ culture in $\alpha$-MEM medium containing $10 \%$ FBS, 1 , 25-dihydroxyvitamin $\mathrm{D}_{3}$ $(10 \mathrm{nM})$, and dexamethasone $(100 \mathrm{nM})$, cells were treated with or without I, C, B, and their combination for 12 days. Dental slices were treated with ultrasonic waves in $1 \mathrm{~mol} / \mathrm{L} \mathrm{NH}_{4} \mathrm{OH}$ to remove adherent cells and stained with $0.1 \%$ toluidine blue solution. Resorption pits were observed 
TABLE 1: Effects of I, C, B, and their combination on bone mineral content (BMC) and bone mineral density (BMD) in OVX mice $(n=10)$.

\begin{tabular}{|c|c|c|c|c|c|c|}
\hline \multirow{2}{*}{ Groups } & \multicolumn{3}{|c|}{$\mathrm{BMC}(\mathrm{mg} / \mathrm{mm})$} & \multicolumn{3}{|c|}{$\operatorname{BMD}\left(\mathrm{mg} / \mathrm{cm}^{3}\right)$} \\
\hline & Total & Trabecular & Cortical & Total & Trabecular & Cortical \\
\hline Sham control & $1.77 \pm 0.27$ & $0.45 \pm 0.13$ & $0.89 \pm 0.17$ & $500 \pm 23$ & $220 \pm 25$ & $898 \pm 19$ \\
\hline OVX control & $1.56 \pm 0.13$ & $0.33 \pm 0.08^{\Delta \Delta \Delta}$ & $0.82 \pm 0.13$ & $426 \pm 20^{\Delta \Delta \Delta}$ & $114 \pm 16^{\Delta \Delta \Delta}$ & $910 \pm 19$ \\
\hline Nylestriol & $1.86 \pm 0.21^{*}$ & $0.43 \pm 0.04^{* *}$ & $0.78 \pm 0.14$ & $508 \pm 29 * * *$ & $205 \pm 20^{* * *}$ & $916 \pm 28$ \\
\hline OVX + I (40 mg/kg) & $1.67 \pm 0.09$ & $0.40 \pm 0.08^{* *}$ & $0.81 \pm 0.16$ & $449 \pm 31^{*}$ & $157 \pm 25^{* *}$ & $906 \pm 20$ \\
\hline OVX + C (20 mg/kg) & $1.62 \pm 0.07$ & $0.37 \pm 0.09^{*}$ & $0.79 \pm 0.12$ & $466 \pm 34^{* *}$ & $162 \pm 30^{* *}$ & $885 \pm 19$ \\
\hline OVX + B (120 mg/kg) & $1.56 \pm 0.12$ & $0.38 \pm 0.11^{*}$ & $0.86 \pm 0.15$ & $437 \pm 45$ & $149 \pm 27^{* *}$ & $881 \pm 26$ \\
\hline $\mathrm{OVX}+\mathrm{I}(40 \mathrm{mg} / \mathrm{kg})+\mathrm{C}(20 \mathrm{mg} / \mathrm{kg})+\mathrm{B}(120 \mathrm{mg} / \mathrm{kg})$ & $1.52 \pm 0.20$ & $0.42 \pm 0.05^{* *}$ & $0.92 \pm 0.17$ & $482 \pm 25^{* *}$ & $195 \pm 22^{* *}$ & $889 \pm 19$ \\
\hline
\end{tabular}

All values are expressed as mean $\pm \mathrm{SD},{ }^{\triangle \Delta \Delta} P<0.001$ versus sham group; ${ }^{*} P<0.05,{ }^{* *} P<0.01,{ }^{* * *} P<0.001$ versus OVX group.

under a microscope; 20 vision fields of each dental slice were randomly chosen to measure the pit area with image analysis software (Leica Q550IW, Germany). The sum of the resorption pit area of 20 random vision fields was calculated as resorption pit area of each dental slice. The inhibitory effects of the tested compounds on bone resorption were expressed as the resorption pit area of dental slice treated with test compounds/resorption pit area of control $\times 100 \%$.

\subsection{Assay for Cytoskeleton of Osteoclasts}

2.4.1. Osteoclasts Induced with M-CSF and RANKL from Bone Marrow Cells. $5 \times 10^{7}$ bone marrow cells were cultured in a 6-well plate containing $\alpha$-MEM supplemented with 10\% FBS and $5 \mathrm{ng} / \mathrm{mL} \mathrm{M}-\mathrm{CSF}$ in a humidified atmosphere of $5 \% \mathrm{CO}_{2}$ for 24 hours. Nonadherent cells were collected and cultured in $\alpha$-MEM medium containing $10 \%$ FBS and $50 \mathrm{ng} / \mathrm{mL} \mathrm{M-}$ CSF for 3 days. Cells remaining on the bottom of the wells were considered as bone marrow-derived macrophages and cultured in $\alpha$-MEM containing 10\% FBS, $50 \mathrm{ng} / \mathrm{mL}$ M-CSF, and $100 \mathrm{ng} / \mathrm{mL}$ RANKL. The culture medium was replaced with fresh medium every 3 days, and after 6 days, the cells differentiated into mature osteoclasts. The osteoclasts were used to investigate the formation of actin ring and to analyze the expression of regulating proteins.

2.4.2. Assay for Formation of Actin Ring. Osteoclastic cells (1 $\left.\times 10^{6}\right)$ induced with M-CSF and RANKL were seeded onto the $10 \times 10 \mathrm{~mm}$ glass coverslips and treated with or without $\mathrm{I}$, $\mathrm{C}, \mathrm{B}$, and their combination for $4 \mathrm{~h}$. The osteoclasts were then fixed with $4 \%$ fresh paraformaldehyde in PBS for $15 \mathrm{~min}$, washed three times with PBS, and then permeabilized with $0.1 \%$ Triton X-100 in PBS for $10 \mathrm{~min}$. F-actin ring in the cells was labeled with rhodamine-conjugated phalloidin by incubating for $30 \mathrm{~min}$ in darkness. Nuclei were stained at $37^{\circ} \mathrm{C}$ for $10 \mathrm{~min}$ with $5 \mu \mathrm{g} / \mathrm{mL}$ Hoechst 33258 solution, and after further washes in PBS, the cells were mounted in glycerol. Specimens were observed using a confocal laser scanning microscope (LEICA TCS-SP5, Germany) with appropriate combinations of filters and mirrors.

2.5. Western Blot. Cells from neonatal rat cavarial osteoblasts or osteoclasts induced with M-CSF and RANKL from bone marrow cells (as described in Section 2.4.1) were treated with or without I, C, B, and their combination for $24 \mathrm{~h}$. Cells were lysed in a buffer containing $20 \mathrm{mM}$ Tris- $\mathrm{HCl}$, $150 \mathrm{mM} \mathrm{NaCl}, 1 \%$ Triton X-100, and protease and phosphatase inhibitors. The lysates (30-40 mg) were separated by $10 \%$ SDS PAGE and transferred to a polyvinylidene difluoride membrane. After blocking with 5\% skim milk, the membrane was probed with anti-OPG and RANKL for osteoblasts or anti-phospho Akt, ERK, JNK, p38, and I $\kappa$ $B$ for osteoclasts. The same membrane was stripped and reprobed and chemiluminescent signals were detected with a Gel Doc 2000 luminescent image analyzer (Wealtec DolphinDoc, USA).

2.6. Statistical Analysis. The experiments were repeated three times in five replicate samples. Data were expressed as mean \pm standard deviation and one-way ANOVA, followed by Dunnett's $t$-test which was used for statistical analysis (PASW 18.0 software; SPSS Inc., Chicago, USA), and the level of significance was set at $P<0.05\left(^{*}\right), P<0.01\left(^{* *}\right), P<0.001$ $(* * *)$.

To determine if the compounds were acting synergistically, we used the probability sum test ( $q$ test) $[19,20]$. The formula used is as follows: $q=E_{\mathrm{A}+\mathrm{B}} /\left(E_{\mathrm{A}}+E_{\mathrm{B}}-E_{\mathrm{A}} \times\right.$ $\left.E_{\mathrm{B}}\right)$. Here, $\mathrm{A}$ and $\mathrm{B}$ indicate compound $\mathrm{A}$ and compound $\mathrm{B} ; E$ is the rate of change in the treated group compared with the mean values in the control group. $E_{\mathrm{A}+\mathrm{B}}$ is the real percentage of responders and $\left(E_{\mathrm{A}}+E_{\mathrm{B}}-E_{\mathrm{A}} \times E_{\mathrm{B}}\right)$ is the expected response rate. $\left(E_{\mathrm{A}}+E_{\mathrm{B}}\right)$ is the sum of the probabilities when compound $\mathrm{A}$ and compound $\mathrm{B}$ are used alone. $\left(E_{\mathrm{A}} \times E_{\mathrm{B}}\right)$ is the probability of cells responding to both compounds when they were used alone. When $q$ was $<0.85$, the combination was thought to be antagonistic; when $q>1.15$, the combination was thought to be synergistic; when $q$ was between 0.85 and 1.15 , the combination was thought to be additive.

\section{Results}

3.1. ICB Decrease Bone Loss in Ovariectomized Osteoporotic Mice. As shown in Table 1, 12 weeks after ovariectomy, tibia bone mineral content (BMC) and bone mineral density (BMD) significantly decreased in total and trabecular bone compared with sham mice, but did not change in cortical bone. Administration of nylestriol significantly increased 
total and trabecular bone BMC and BMD in tibia compared to the OVX control. Administration of I, C, B, and their combination significantly increased BMC and BMD of trabecular bone and total BMD, but did not change total BMC in tibia. The effects of their combination were more potent than the individual compounds. These results indicate that I, C, B, and their combination improve $\mathrm{BMC}$ and $\mathrm{BMD}$ of trabecular bone and decrease bone loss induced by ovariectomy.

As shown in Figure 1, administration of I, C, B and their combination did not cause the increase of uterine weights and inhibit weight gain of ovariectomized mice, except for icariin which inhibited the body weights gain. Serum deoxypyridinoline cross-links to creatinine ratio (DPD/Cr), TRAP levels are biochemical markers of bone resorption, and ALP is a marker for bone formation. Ovariectomy induced high bone turnover in mice as evidenced by a significant increase in serum DPD/Cr, TRAP, and ALP levels. Nylestriol decreased serum DPD/Cr, TRAP, and ALP levels and inhibited high bone turnover in OVX mice. Administration of I, $\mathrm{C}, \mathrm{B}$, and their combination decreased serum DPD/Cr and TRAP levels, but no decrease in serum ALP levels. OPG (osteoprotegerin) is an endogenous protein produced by osteoblastic cells, which inhibits osteoclast formation and activation. Ovariectomy decreased serum OPG levels and in contrast, nylestriol increased serum OPG. The I, C, B, and their combination significantly increased OPG levels in ovariectomized mice. These results show that I, C, B, and their combination increased bone density by inhibiting bone resorption, and their combination showed higher activity than the individual compounds. Therefore, the effects and interaction of I, C, and B were further investigated for their mechanism of action and mutual interaction on osteoclastic bone resorption.

3.2. ICB Synergistically Inhibits Osteoclast Formation and Differentiation. To clarify the effect of I, C, and B alone or their combination on osteoclast formation, bone marrow cells were cocultured with osteoblasts derived from rat calvaria in the presence of $10^{-8} \mathrm{M} 1,25$-dihydroxyvitamin $\mathrm{D}_{3}$. Many TRAP-positive osteoclasts were formed in the coculture system within 6 days in response to $1 \alpha, 25(\mathrm{OH})_{2} \mathrm{D}_{3}$. As shown in Figures 2(a) and 2(b), the numbers of TRAPpositive multinucleated cells were significantly reduced by I, $\mathrm{C}$, and $\mathrm{B}$ or their combinations. In treatment of osteoclast with I, C, or B alone, the numbers of TRAP-positive multinucleated cells were reduced to $68.1 \%, 70.7 \%$, and $63.5 \%$ of control, respectively. The combination of IB, IC, $\mathrm{BC}$, and IBC decreased the number of osteoclasts to $37.1 \%$, $53.0 \%, 46.0 \%$, and $21.4 \%$ of control, respectively. According to the $q$ value, the combination of IC, BC, and IB exerted additive inhibitory effects, and the combination of ICB exerted somewhat synergistic inhibitory effects on osteoclast formation.

TRAP activity was directly related with osteoclastic bone resorption and as shown in Figure 2(c), after 48-h treatment, TRAP activity was significantly suppressed by I, B, C, and their combination. In treatment of osteoclast with I, B, and $\mathrm{C}$ alone, the TRAP activity decreased to $89.4 \%, 80.9 \%$ and $90.4 \%$ of control, respectively. The combination of IB, IC, BC, and IBC reduced the TRAP activity to $74.8 \%$, $77.6 \%, 74.9 \%$, and $60.2 \%$ of control, respectively. According to the $q$ value, the combination of IB and BC exerted additive inhibitory effects; the combination of IC and ICB exerted synergistic inhibitory effects on the TRAP activity of osteoclast.

Another possible mechanism of the inhibitory effect on bone resorption of mature osteoclasts is that I, B, C, and their combination could reduce bone matrix degradation by inhibiting cathepsin K (CK) activity [21]; hence, we further evaluated the effects on cathepsin $\mathrm{K}$ activity of osteoclasts in a cell-free enzyme assay using synthetic substrate Ac-LRAFC and recombinant cathepsin K. As shown in Figure 2(d), I, $\mathrm{B}$, and $\mathrm{C}$ inhibited the osteoclastic CK activity to $86.4 \%$, $88.9 \%$, and $86.6 \%$ of control, respectively. Although the treatment of combinations of I, C, and B performed more potential suppressive effects than individual compounds, these combinations exerted antagonistic or additive, but not synergistic, inhibitory effects on cathepsin $\mathrm{K}$ activity of osteoclast according to the $q$ value.

\subsection{ICB Synergistically Suppresses Osteoclastic Bone Resorp-} tion. The dental slices were cocultured with osteoclast for 12 days. Untreated dental slices possess a very homogenous surface. Mature osteoclasts erode this homogenous surface and form resorption pits. After staining with toluidine blue, the resorption pits can be identified easily by their blue color. Under the experimental condition, a statistically significant reduction of number and area of bone resorption pit were observed on the dental slices treated with I, C, B, and their combination compared with control. In the mono-treatment group, I, C, or B alone decreased the area of bone resorption pit to $65.2 \%, 67.5 \%$, and $62.4 \%$ of control. Bitreatment of I, $\mathrm{C}$, and $\mathrm{B}$ decreased the area of bone resorption pit to $33.9 \%, 36.8 \%$, and $39.4 \%$ of control. The ICB combination treatment decreased the area of bone resorption pit to $12.8 \%$ of control (Figures 3(a) and 3(b)). According to the $q$ value, the combination of I, C, and B exerted slightly synergistic inhibitory effects on the bone resorption of osteoclast. These results suggested that ICB combination intensifies the inhibitory effect on osteoclastic bone resorption.

\subsection{ICB Synergistically Blocks RANKL-Induced Osteoclasts Cytoskeletal Organization. Osteoclastic bone resorption is initiated by the attachment of osteoclasts to the bone surface. Upon attachment, the osteoclasts form characteristic actin rings, a cytoskeletal structure essential for optimal osteoclastic bone resorption [22]. To determine if ICB affects cytoskeletal organization in osteoclasts, bone marrow cells were cultured on glass coverlips in the presence of M-CSF and RANKL with or without I, C, B, and their combination. The actin rings were visualized by staining with rhodamine- conjugated phalloidin to assess the activity in the early phase of bone resorption. As shown in Figure 4, confocal laser scanning microscopy revealed that actin rings were dense and had some pseudopodium lying in their periphery. Treatment of osteoclasts with I, C, B, and their combination caused}



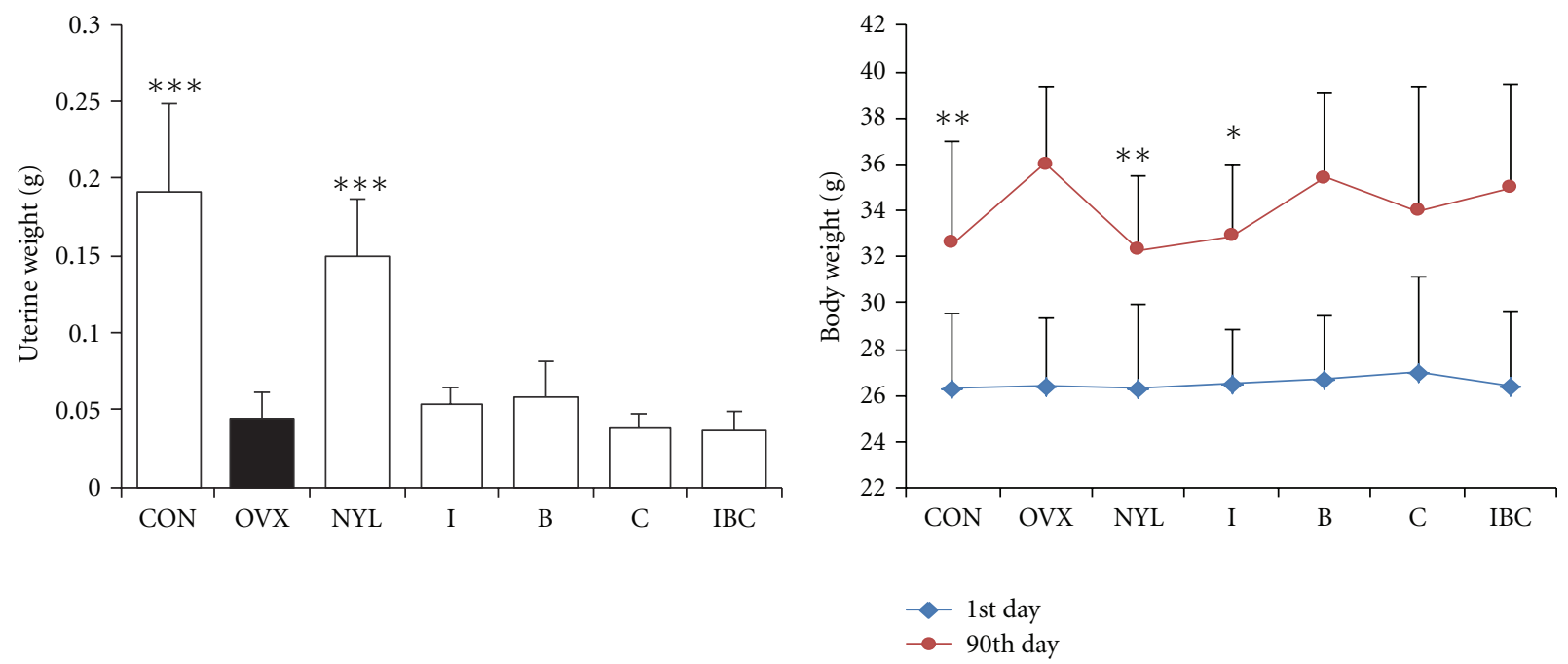

(a)

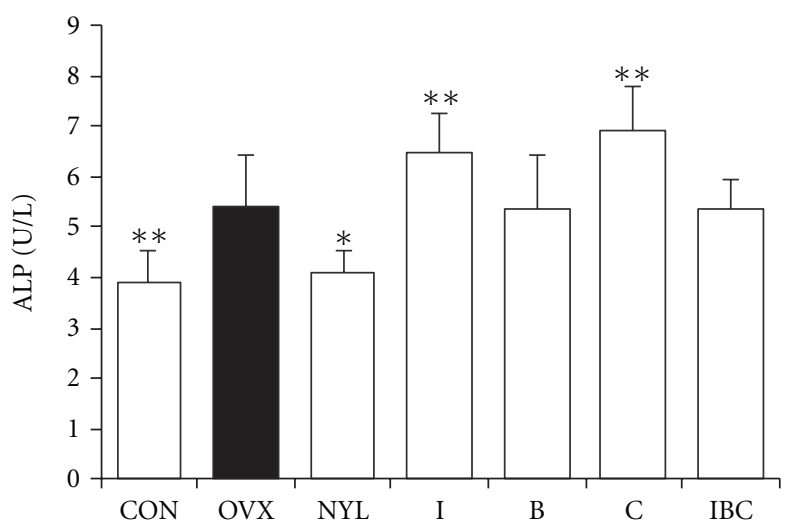

(c)

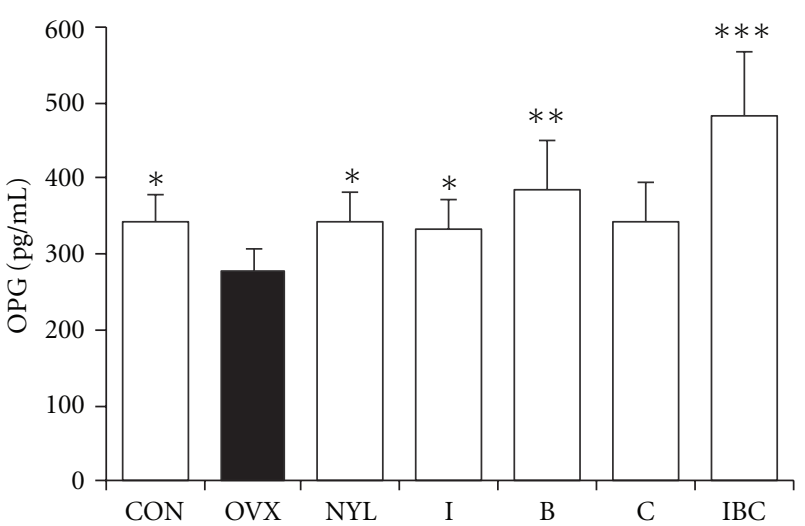

(d)

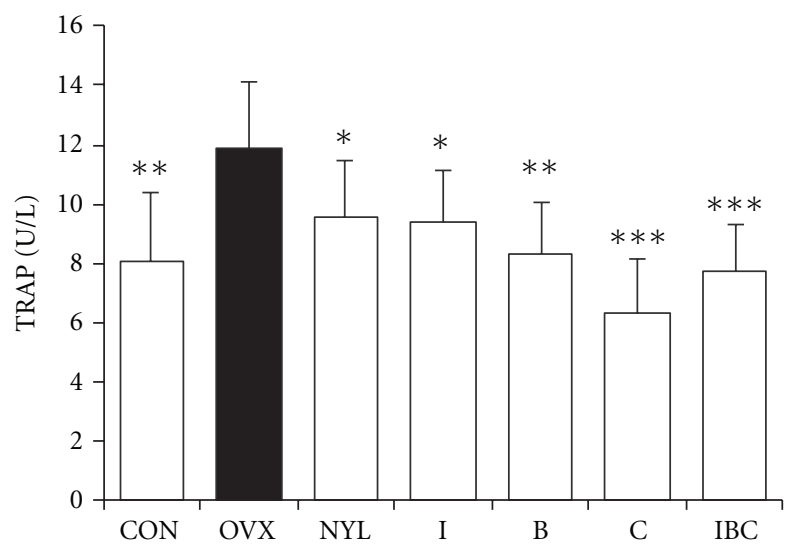

(e)

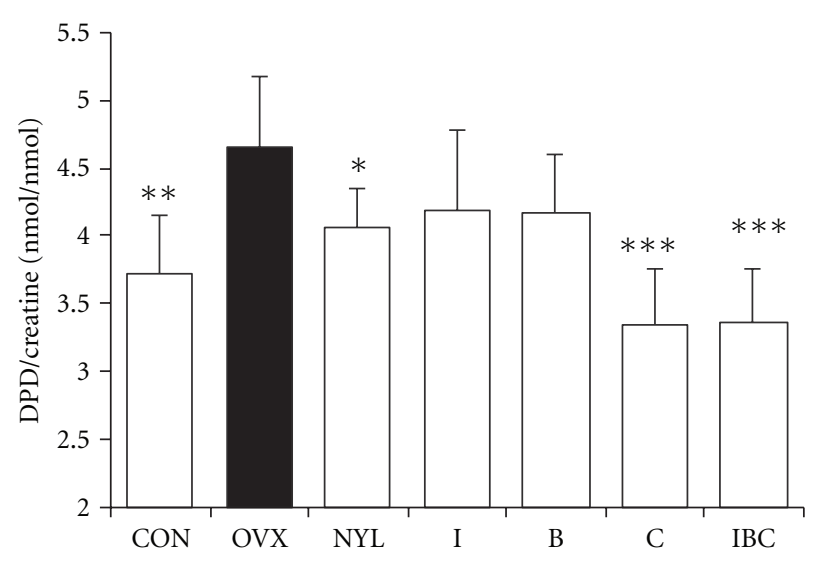

(f)

Figure 1: Effects of I, B, C, and their combination on ovariectomized osteoporotic mice. Ovariectomized mice were administered with I ( $40 \mathrm{mg} / \mathrm{kg}), \mathrm{B}(120 \mathrm{mg} / \mathrm{kg}), \mathrm{C}$ (20 mg/kg), and their combination (I $40 \mathrm{mg} / \mathrm{kg}+\mathrm{C} 20 \mathrm{mg} / \mathrm{kg}+\mathrm{B} 120 \mathrm{mg} / \mathrm{kg})$ for $12 \mathrm{weeks}$. Then, the uterine and body weight were weighed, the serum biochemical parameters were assayed. (a) uterine weight; (b) body weight; (c) ALP activity; (d) OPG content; (e) TRAP activity; (f) DPD/creatine. Data were presented as mean \pm standard deviation, $(n=10) .{ }^{*} P<0.05$, and ${ }^{* *} P<0.01$, *** $P<0.001$ compared with OVX control. 


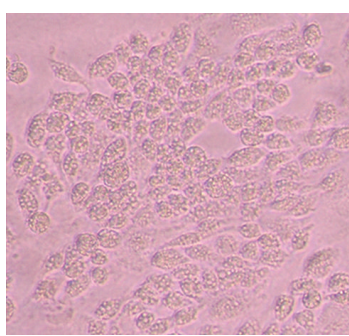

CON

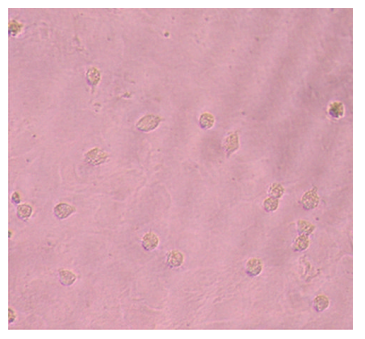

IB

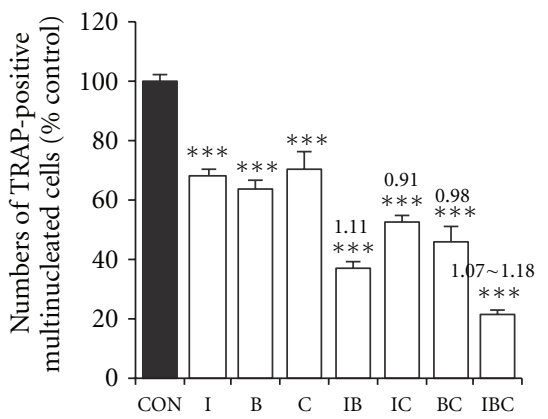

(b)

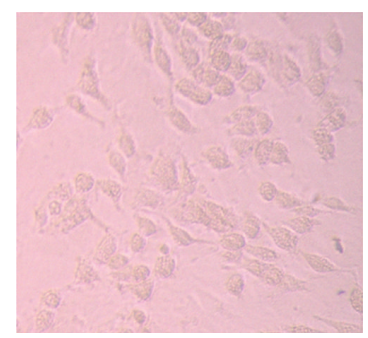

I

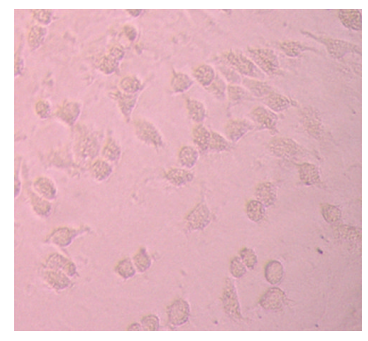

IC

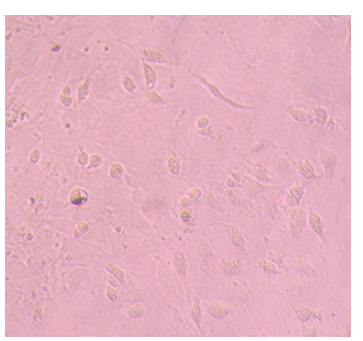

B

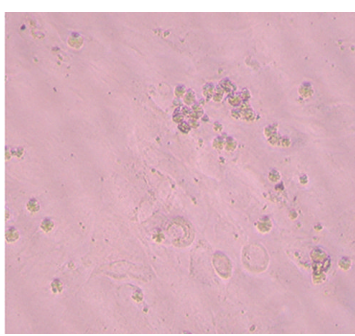

BC

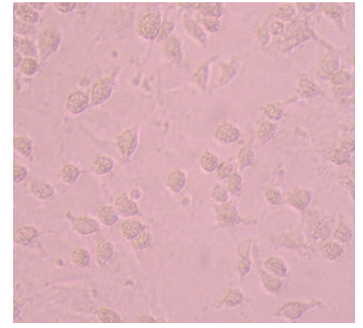

C

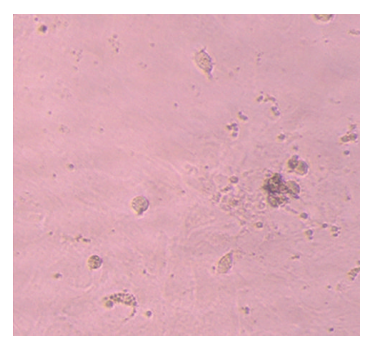

IBC

(a)

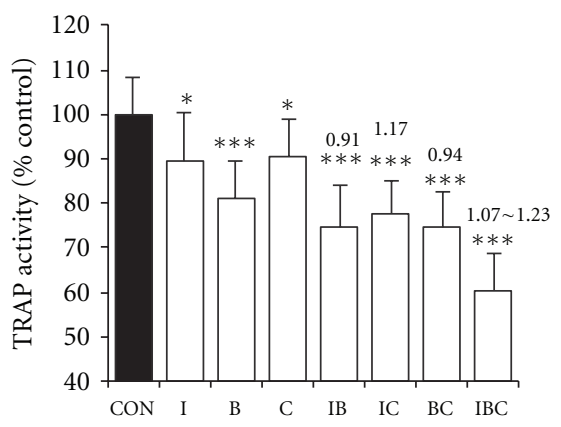

(c)

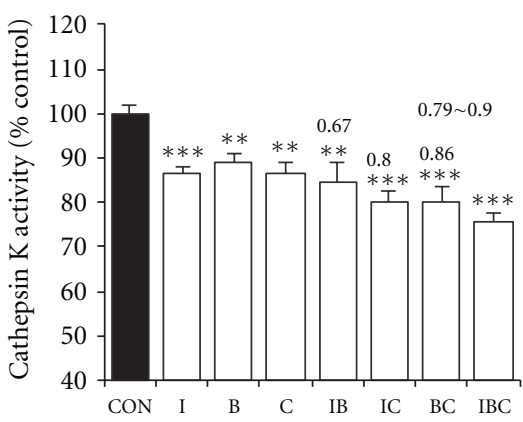

(d)

FIgURE 2: Inhibitory effects of I, B, C, and their combination on osteoclasts. (a) The morphological change of osteoclast $(\times 200)$. Osteoclasts in the coculture system of primary osteoblasts and bone marrow cells were cultured for $24 \mathrm{~h}$ and then treated with or without the I, B, C, and their combination for 10 days. (b) Osteoclasts were treated for 10 days and then stained for TRAP, and those possessing three or more nuclei were counted as osteoclast-like multinucleated osteoclasts under a microscope. (c) Osteoclasts in the coculture system of primary osteoblasts and bone marrow cells were cultured for 6 days and then treated with or without I, B, C, and their combination for 48 hours. TRAP activity was measured by $p$-nitrophenyl sodium phosphate assay. (d) Cells were treated as described in TRAP assay, and the activity of cathepsin K was measured by a fluorometer equipped with a $400 \mathrm{~nm}$ excitation filter and $505 \mathrm{~nm}$ emission filter. Data were presented as mean \pm standard deviation. The experiments were repeated 3 times in five replicate samples $(n=5) * P<0.05,{ }^{* *} P<0.01$, ${ }^{* * *} P<0.001$ compared with control. The number on the column is $q$ value, indicating the interaction between $\mathrm{I}, \mathrm{B}$, and C.

pseudopodium to vanish; F-actin ring was disrupted and was loose and thin in the cytoplasmic area of osteoclasts. The effects of the combination of I, C, and B on the formation of F-actin ring are more potent than that of individual compounds, indicating that I, C, and B could synergistically affect cytoskeletal organization, which is necessary for bone resorption by osteoclasts.

\subsection{ICB Elevates the Ratio of OPG and RANKL Expression in} Osteoblast. In coculture system, osteoblasts support the differentiation of osteoclast progenitors by expressing RANKL in response to vitamin $\mathrm{D}_{3}$ and dexamethasone [23]. Thus, it is necessary to determine whether these antiosteoclastogenic compounds were directly affecting osteoclast precursor cells or indirectly targeting to osteoblast and thus modulating the expression of OPG and RANKL, which are critical factors for osteoclast differentiation and activity. In treatment of osteoblast with I, C, B, and their combination for $24 \mathrm{~h}$, the relative protein expressions of OPG in osteoblast were enhanced to 2.38-, 2.42-, 4.13-, 3.29-, 2.11-, 1.18-, and 1.32fold of control, respectively; the relative protein expressions of RANKL were 1.09-, 1.06-, 1.14-, 0.72-, 0.53-, 0.22-, and 0.39 -fold of control, respectively. Therefore, the ratios of the protein expression levels of OPG and RANKL were up to 2.17-, 2.29-, 3.62-, 4.56-, 3.98-, 5.37-, and 3.36-fold 


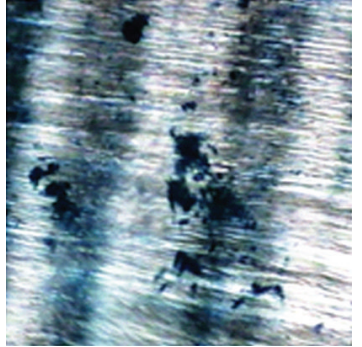

CON

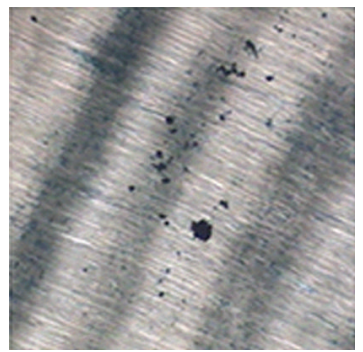

IB

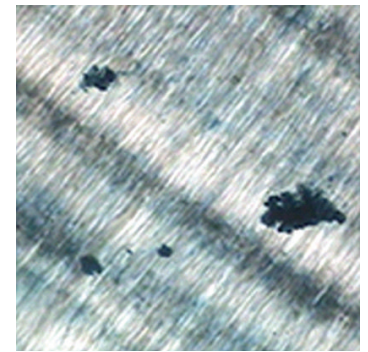

I

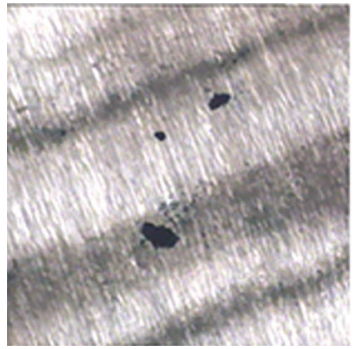

IC

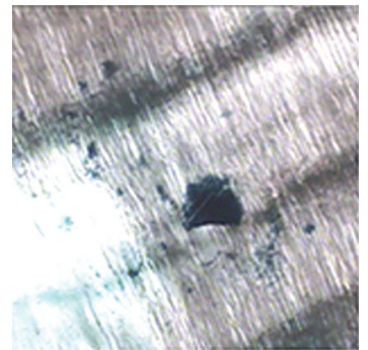

B

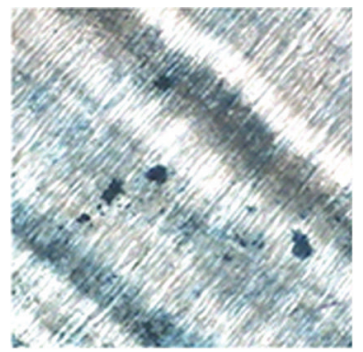

$\mathrm{BC}$

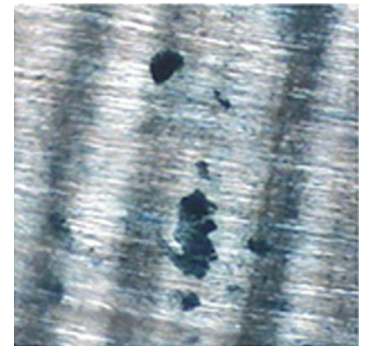

C

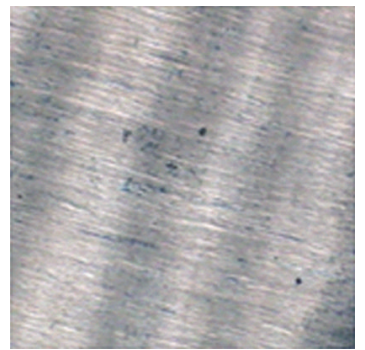

IBC

(a)

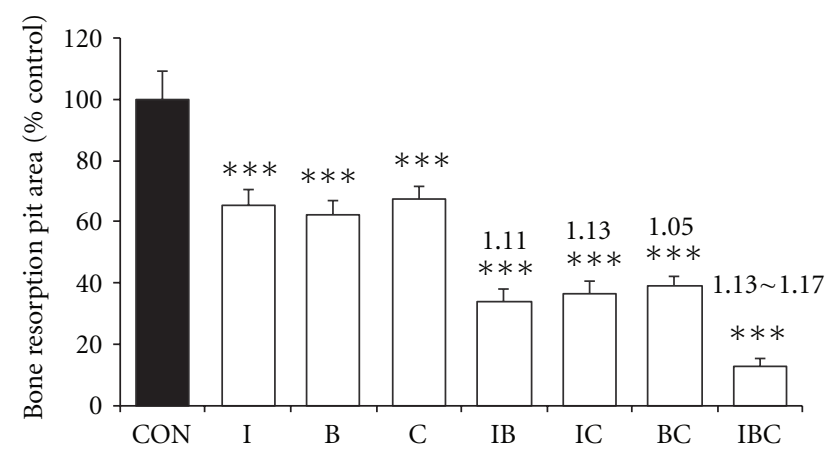

(b)

Figure 3: Inhibitory effects of I, B, C, and their combination on osteoclastic bone resorption. Primary osteoblasts and bone marrow cells were cocultured with a sterilized dental slice in $\alpha$-MEM medium in the presence of 1,25 -dihydroxyvitamin $\mathrm{D}_{3}(10 \mathrm{nM})$ and dexamethasone $(100 \mathrm{nM})$ and treated with or without I, B, C, and their combination for 12 days. Resorption pits were observed under a microscope, and the pit area was quantitated with image analysis software. (a) Bone resorption pit on dental slices $(\times 100)$; (b) the change of bone resorption pit area under the treatment of I, B, C, and their combination. Data were presented as mean \pm standard deviation. The experiments were repeated 3 times in five replicate samples $(n=5)$. ${ }^{*} P<0.05,{ }^{* *} P<0.01,{ }^{* * *} P<0.001$ compared with control. The number on the column is $q$ value, indicating the interaction between $\mathrm{I}, \mathrm{B}$, and $\mathrm{C}$.

of control, respectively (Figure 5). These results indicated that I, B, C, and their combinations could regulate the OPG/RANKL system in osteoblast, resulting in the inhibition of osteoclast differentiation in a coculture model system.

3.6. ICB Causes the Decrease of Phosphorylation of RANK Signaling to MAPKs Pathways. RANKL stimulation increases phosphorylation of Akt, ERK, JNK, and p38 in osteoclasts, and activation of the NF- $\kappa \mathrm{B}$ pathway requires the phosphorylation of I- $\kappa \mathrm{B}$ [24]. Thus, we next determined whether I, C, B, and their combination could affect the signaling pathways involving these kinases. As shown in Figure 6, treatment of osteoclasts with I, C, and $\mathrm{B}$ alone, the phosphorylation of $\mathrm{I} \kappa \mathrm{B}$ and ERK were downregulated, and their combination showed more potent inhibitory effects than the individual compounds. Use of I and C alone upregulated the phosphorylation of $\mathrm{p} 38$, and the combination of IB, BC, and IBC significantly decreased this kinase; $B$ increased the phosphorylation of Akt and JNK; all the combination of B with I and C reduced the phosphorylation of Akt and JNK. These results indicated that ICB synergistically decrease the phosphorylation of RANKL signaling in MAPK pathways, leading to the reduction of osteoclastic bone resorption. Taken together, our findings suggested the rationality of EXD in treating osteoporosis.

\section{Discussions}

In this study, we found that, in ovariectomized mice, combined use of the active components of EXD, namely, 


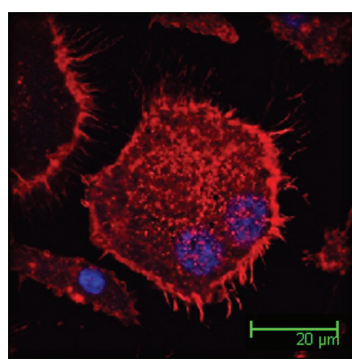

$\mathrm{CON}$

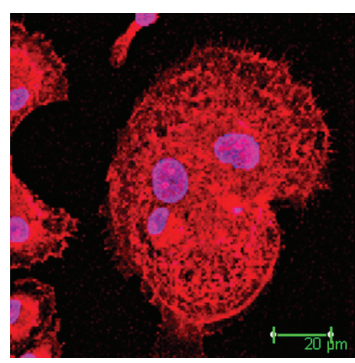

IB
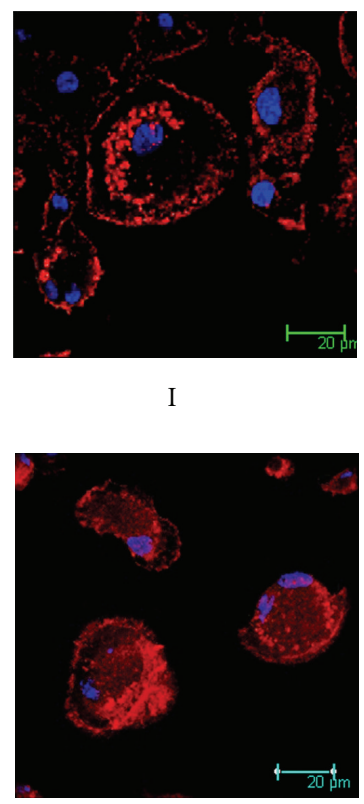

IC

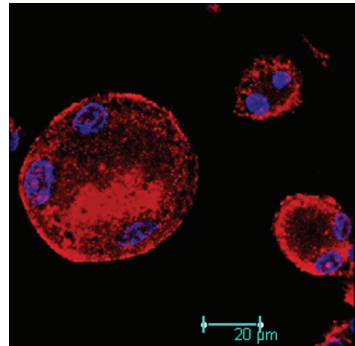

B

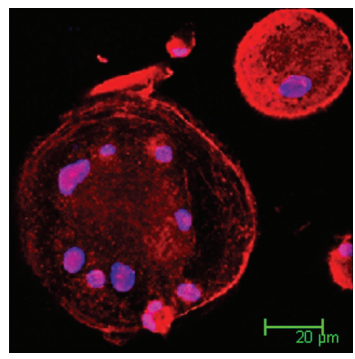

BC

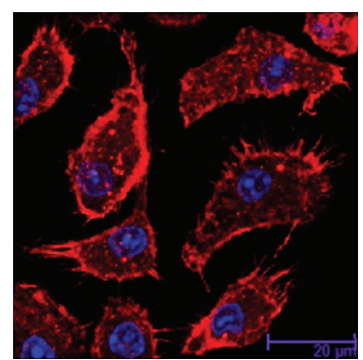

C

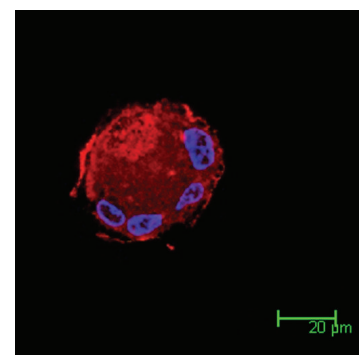

IBC

Figure 4: Effects of I, B, C, and their combination on F-actin rings. Osteoclasts were cultured on glass coverslip for $4 \mathrm{~h}$ in the absence (control) or presence of I, B, C, and their combination. The cells were then fixed and labeled with rhodamine-conjugated phalloidin and Hoechst 33258 and observed under confocal laser scanning microscope.

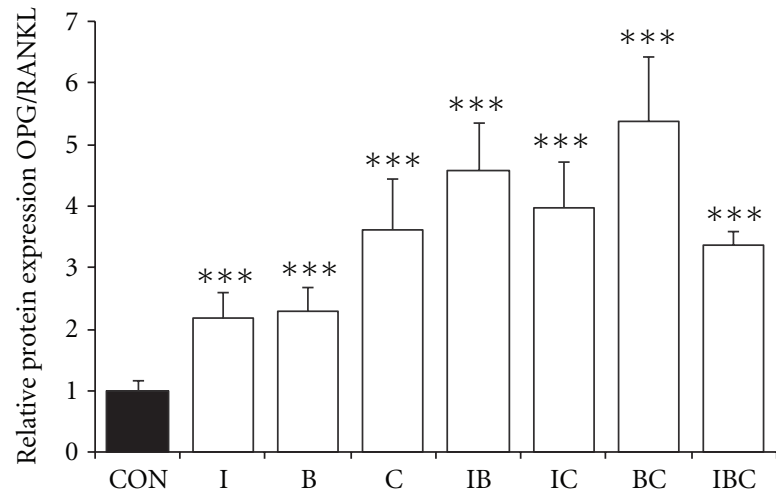

(a)

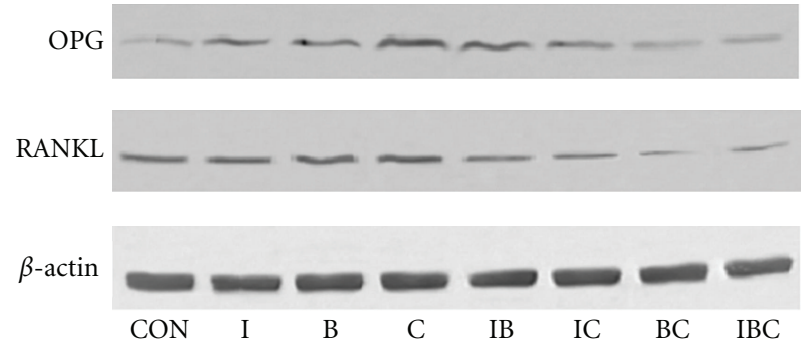

(b)

Figure 5: Effects of I, B, C, and their combination on expression of OPG and RANKL of osteoblasts. Primary osteoblastic cells from neonatal rat calvaria were treated with or without I, B, C, and their combination for $24 \mathrm{~h}$. (a) The ratio of protein expression of OPG and RANKL. (b) Protein expression of OPG and RANKL was determined by Western blotting. $\beta$-actin was used as an internal reference. Data were presented as mean \pm standard deviation. The experiments were repeated 3 times in five replicate samples $(n=5),{ }^{*} P<0.05,{ }^{* *} P<0.01,{ }^{* * *} P<0.001$ compared with control.

icariin (I), berberine (B), and curculigoside (C), significantly increased the bone mineral density and bone mineral content and regulated the serum biochemical parameters. At the cellular level, the IBC inhibited the formation, differentiation, and bone resorption of osteoclast through modulating OPG/RANKL system in osteoblast and MAPKs pathways in osteoclast. Assessment of the $q$ value by the probability sum test directly demonstrated the synergic effect of I, B, and C on osteoclastic cells to some extent. These results clearly show that components of EXD exert effects of mutual reinforcement. However, IBC does not show an intensified adverse effect in the ovariectomized murine model, as revealed by change in body and uterine weight, consistent with the safety of EXD. These observations are in agreement with the rationality of the formula used in this study, mutual reinforcement of the compounds, and reduction of adverse effects.

Osteoclastic bone resorption is mediated by the formation of new osteoclasts and the bone-resorbing activity of osteoclasts. The mature osteoclasts are characterized by multinuclearity, TRAP staining, cathepsin $\mathrm{K}$ activity, an actin ring structure, ruffled border, and acidic cell condition 


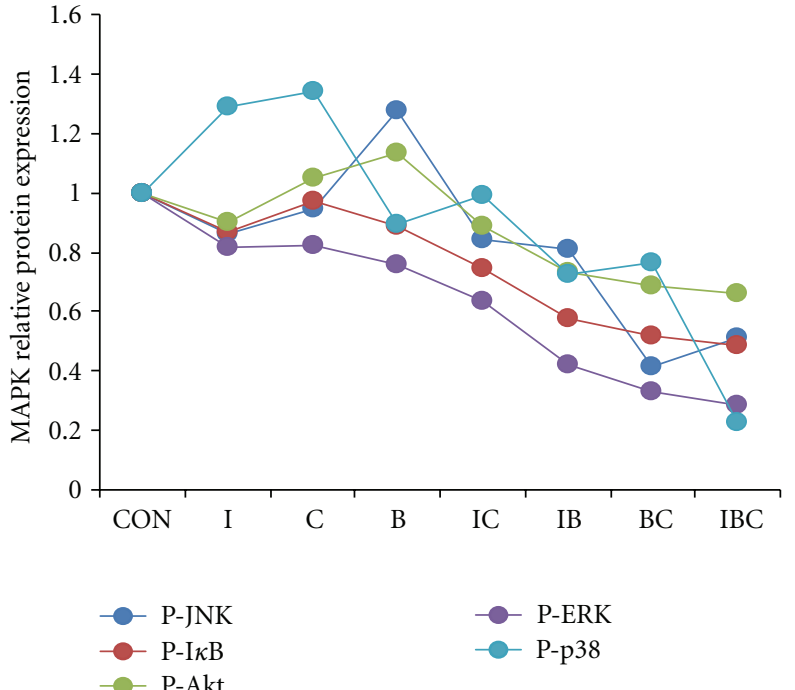

(a)

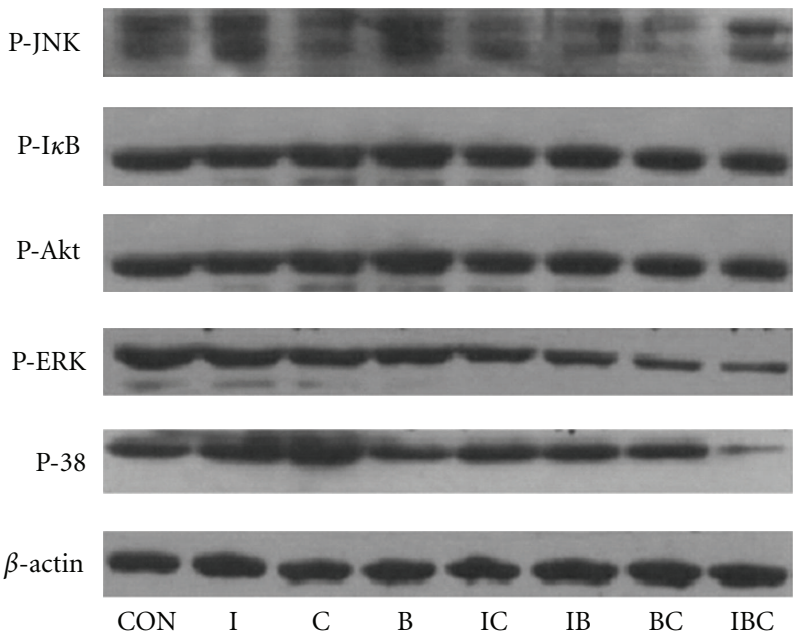

(b)

Figure 6: Effects of I, C, B, and their combination on RANKL-induced signaling pathways. Osteoclasts induced from bone marrow cells by $\mathrm{M}-\mathrm{CSF}$ and RANKL were treated for $24 \mathrm{~h}$ and assessed for phosphorylation of JNK, ERK, p38 MAPK, and Akt and the degradation of I $\kappa \mathrm{B} \alpha$ by Western blot. $\beta$-actin was used as an internal reference.

during resorption [13]. As depicted in Figure 2, exposure of osteoclasts to I, B, C, and their combination significantly reduced the number of TRAP-positive multinucleated cells and the activity of TRAP and cathepsin $\mathrm{K}$ and induced the disruption of actin rings, indicating that $\mathrm{I}, \mathrm{B}, \mathrm{C}$, and their combinations reduced the pit-forming activity of osteoclasts, both by triggering the direct disruption of actin rings and by inhibiting osteoclast formation and the survival of mature osteoclasts. Icariin, which exhibits the strongest therapeutic effects in the ovariectomized osteoporotic rats, has also been shown to be the principal ingredient of EXD in targeting osteoclastic bone resorption [16]; the B and C enhance the inhibitory effects of I on osteoclastic formation, differentiation, and bone resorption, augmenting the effects of I. Thus, the EXD formula proves its rational by modern biochemical analysis.

The receptor activators of NF- $\kappa \mathrm{B}$ ligand (RANKL) and osteoprotegerin (OPG) synthesized by osteoblast plays an essential role in the osteoclastic formation, differentiation, and bone resorbing activity [7]. RANKL provides a signal to osteoclast progenitors through the receptor activator of $\mathrm{NF}-\kappa \mathrm{B}$ (RANK) to activate osteoclast differentiation and function. OPG blocks the interaction between RANKL and the RANK receptor. In other words, OPG inhibits osteoclastogenesis while RANKL supports bone resorption of osteoclast [25]. Therefore, bone remodeling can be assessed by the relative ratio of OPG to RANKL. In the present study, I, B, C, and their combinations significantly increased the relative ratio of OPG to RANKL, indicating that they inhibited the bone resorption by modulating the expression of OPG and RANKL of osteoblast.

The RANK signaling by the interaction with RANKL induces recruitment and activation of tumor necrosis factor receptor-associated factors (TRAFs), leading to the activation of MAPK pathway, including ERK, p38, and JNK [9]. The ERK pathway is involved in the negative regulation of osteoclastogenesis [6]. However, the p38 and JNK pathways have been shown to play a critical role during RANKLinduced osteoclast differentiation [26]. Osteoclast apoptosis is controlled by several signaling molecules, including TRAF6, Src, PI3 K/Akt, ERK, and NF- $\kappa$ B pathways [27]. It was found in our study that the phosporylation of ERK, Akt, JNK, and I $\kappa$ B was decreased slightly and that of osteoclast p38 was increased after I administration; the phosphorylation of ERK was downregulated after C or $\mathrm{B}$ administration alone; the phosphorylation of ERK, Akt, p38, JNK, and I- $\kappa$ B of osteoclasts was downregulated significantly after combined administration of I with $\mathrm{C}$ or/and B. These results suggest that I, C, and B had different modulating mechanisms through MAPK pathways and their combinations had more potent inhibitory effects on the phosphorylation of these signaling protein of osteoclast.

It is worth pointing out that Chinese medicinal formula may have complicated changes by combining various herbs or their constituents. Some may reinforce or decrease their effects, moderate or eliminate their original toxic side effects; some may facilitate the delivery of the principle element to the disease site in the body [28]. In EXD, Epimedii Folium and Curculiginis Rhizoma have similar properties and effects their combination could reinforce each other's action. Phellodendri Chinensis Cortex has different pharmacological action from Epimedii Folium and Curculiginis Rhizoma but could assist in raising their therapeutic effects [17]. I, C, and $\mathrm{B}$ are major active constituents from Epimedii Folium, Curculiginis Rhizoma, and Phellodendri Chinensis Cortex, 
respectively. It may be an interesting observation in this work that ICB treatment decreases the osteoclastic bone resorption compared with I treatment alone, whereas I combined with $\mathrm{C}$ and/or B significantly upregulates OPG, and downregulates RANKL. Because OPG was upregulated, RANKL was downregulated $24 \mathrm{~h}$ after ICB treatment; some other events might also be involved in the enhancement of I action by C and $B$ at the earlier stage. Interestingly, the same results were also observed in the MAPKs pathways. The phosporylation of P38, ERK, Akt, JNK, and I $\kappa$ B was decreased slightly after I administration, while the phosphorylation of these signaling proteins was downregulated significantly after combined administration of I with $\mathrm{C}$ or/and $\mathrm{B}$. These results suggest that $\mathrm{C}$ and $\mathrm{B}$ might assist $\mathrm{I}$ in the EXD formula to modulate these key pathways.

The antiosteoporotic activity and mechanism of icariin, curculigoside, and berberine have been extensively studied. Icariin enhances the differentiation and proliferation of osteoblasts and facilitates matrix calcification through the induction of BMP-2, BMP-4, and NO synthesis, subsequently regulating $\mathrm{Cbf} \alpha 1 / \mathrm{Runx} 2, \mathrm{OPG}$, and RANKL gene expressions and activating BMP signaling [29-32]. Icariin also inhibited osteoclastic differentiation and reduced the motility and osteoclastic bone resorption [29, 33]. These regulatory effects of icariin on bone remodeling are also related with estrogen-like activity [30,31]. Curculigoside has definite effects on osteoblast and osteoclast, enhances the expression of vascular endothelial growth factor and bone morphogenetic protein-2 in osteoblastic MC3T3, and prevents hydrogen peroxide-induced dysfunction and oxidative damage in calvaria osteoblasts [34-36]. Berberine inhibits RANKL-mediated osteoclast formation and survival through suppression of NF- $\kappa \mathrm{B}$ and Akt activation [37]. In osteoblastic cells, berberine enhanced the expression of osteogenic marker genes including osteopontin and osteocalcin through activation of Runx2 by p38 MAPK [38]. These findings indicated that icariin, curculigoside, and berberine modulate the bone metabolism through multitargets and pathways. Furthermore, EXD contains various kinds of active components, including flavonoids, phenolic glycoside, alkaloids, anthraquinone, organic acid, and polysaccharide. It is likely that a complicated interaction of active components exists on multiple targets. Therefore, the protective effects of EXD on bone loss are achieved in a similar manner in that multiactive components are exerting their effects through multitargets and pathways; however, these need to be investigated in more depth.

In conclusion, the present study clarifies the interaction relationship on osteoclast between icariin, curculigoside, and berberine. This supports the theory that in TCM formula, the use of multiherbs or their ingredients can provide mutual reinforcement and assistance thus enhancing the therapeutic effects when compared to individual ingredients.

\section{Conflict of Interests}

There is no conflict of interests among the authors.

\section{Authors' Contribution}

L. Xue and L. Jiao contributed equally to this work.

\section{Acknowledgment}

The work was supported by National Natural Science Foundation of China ( 81173191) and Shanghai Committee of Science and Technology, China (12401900702).

\section{References}

[1] K. F. Cheng, K. S. Leung, and P. C. Leung, "Interactions between modern and Chinese medicinal drugs: a general review," American Journal of Chinese Medicine, vol. 31, no. 2, pp. 163-169, 2003.

[2] X. J. Li and H. Y. Zhang, "Synergy in natural medicines: implications for drug discovery," Trends in Pharmacological Sciences, vol. 29, no. 7, pp. 331-332, 2008.

[3] E. M. Williamson, "Synergy and other interactions in phytomedicines," Phytomedicine, vol. 8, no. 5, pp. 401-409, 2001.

[4] Z. Cole, E. Dennison, and C. Cooper, "Update on the treatment of post-menopausal osteoporosis," British Medical Bulletin, vol. 86, no. 1, pp. 129-143, 2008.

[5] M. Błazej and C. Adam, "Selective estrogen receptor modulators in treatment of postmenopausal osteoporosis," Ginekologia Polska, vol. 80, no. 3, pp. 213-217, 2009.

[6] G. D. Roodman, "Advances in bone biology: the osteoclast," Endocrine Reviews, vol. 17, no. 4, pp. 308-332, 1996.

[7] K. T. Steeve, P. Marc, T. Sandrine, H. Dominique, and F. Yannick, "IL-6, RANKL, TNF-alpha/IL-1: interrelations in bone resorption pathophysiology," Cytokine and Growth Factor Reviews, vol. 15, no. 1, pp. 49-60, 2004.

[8] L. G. Raisz, "Pathogenesis of osteoporosis: concepts, conflicts, and prospects," Journal of Clinical Investigation, vol. 115, no. 12, pp. 3318-3325, 2005.

[9] Z. H. Lee and H. H. Kim, "Signal transduction by receptor activator of nuclear factor kappa B in osteoclasts," Biochemical and Biophysical Research Communications, vol. 305, no. 2, pp. 211-214, 2003.

[10] B. R. Wong, D. Besser, N. Kim et al., "TRANCE, a TNF family member, activates Akt/PKB through a signaling complex involving TRAF6 and c-Src," Molecular Cell, vol. 4, no. 6, pp. 1041-1049, 1999.

[11] H. Takayanagi, S. Kim, T. Koga et al., "Induction and activation of the transcription factor NFATc1 (NFAT2) integrate RANKL signaling in terminal differentiation of osteoclasts," Developmental Cell, vol. 3, no. 6, pp. 889-901, 2002.

[12] N. Ishida, K. Hayashi, M. Hoshijima et al., "Large scale gene expression analysis of osteoclastogenesis in vitro and elucidation of NFAT2 as a key regulator," Journal of Biological Chemistry, vol. 277, no. 43, pp. 41147-41156, 2002.

[13] W. J. Boyle, W. S. Simonet, and D. L. Lacey, "Osteoclast differentiation and activation," Nature, vol. 423, no. 6937, pp. 337-342, 2003.

[14] H. Y. Chen, W. C. S. Cho, S. C. W. Sze, and Y. Tong, "Treatment of menopausal symptoms with er-xian decoction: a systematic review," American Journal of Chinese Medicine, vol. 36, no. 2, pp. 233-244, 2008.

[15] H. Nian, L. P. Qin, Q. Y. Zhang, H. C. Zheng, Y. Yu, and B. K. Huang, "Antiosteoporotic activity of Er-Xian Decoction, a traditional Chinese herbal formula, in ovariectomized rats," 
Journal of Ethnopharmacology, vol. 108, no. 1, pp. 96-102, 2006.

[16] L. Qin, T. Han, Q. Zhang et al., "Antiosteoporotic chemical constituents from Er-Xian Decoction, a traditional Chinese herbal formula," Journal of Ethnopharmacology, vol. 118, no. 2, pp. 271-279, 2008.

[17] J. J. Li, J. T. Li, and J. P. Fu, "Erxian Tang-introduction of a Chinese herbal formula, clinical practice, and experimental studies," Chinese Journal of Integrative Medicine, vol. 13, no. 1, pp. 67-73, 2007.

[18] Z. D. Liu, H. S. Zang, and Y. P. Ou-Yang, "Biological study of growth pattern of newborn rat calvaria osteoblastic cell in vitro," Acta Anatomica Sinica, vol. 26, no. 2, pp. 157-159, 1995.

[19] Z. J. Jin, "Addition in drug combination," Acta Pharmacologica Sinica, vol. 1, no. 2, pp. 70-76, 1980.

[20] P. Han, F. M. Shen, H. H. Xie et al., "The combination of atenolol and amlodipine is better than their monotherapy for preventing end-organ damage in different types of hypertension in rats," Journal of Cellular and Molecular Medicine, vol. 13, no. 4, pp. 726-734, 2009.

[21] P. Garnero, O. Borel, I. Byrjalsen et al., "The collagenolytic activity of cathepsin $\mathrm{K}$ is unique among mammalian proteinases," Journal of Biological Chemistry, vol. 273, no. 48, pp. 32347-32352, 1999.

[22] S. L. Teitelbaum, "Osteoclast and its unique cytoskeleton," Annals of the New York Academy of Science, vol. 1240, no. 1, pp. 14-17, 2011.

[23] S. Khosla, "Minireview: the OPG/RANKL/RANK system," Endocrinology, vol. 142, no. 12, pp. 5050-5055, 2001.

[24] M. Matsumoto, T. Sudo, T. Saito, H. Osada, and M. Tsujimoto, "Involvement of p38 mitogen-activated protein kinase signaling pathway in osteoclastogenesis mediated by receptor activator of NF- $\kappa$ B ligand (RANKL)," Journal of Biological Chemistry, vol. 275, no. 40, pp. 31155-31161, 2000.

[25] X. H. Liu, A. Kirschenbaum, S. Yao, and A. C. Levine, "Crosstalk between the interleukin- 6 and prostaglandin E2 signaling systems results in enhancement of osteoclastogenesis through effects on the osteoprotegerin/receptor activator of nuclear factor- $\kappa$ B (RANK) ligand/RANK system," Endocrinology, vol. 146, no. 4, pp. 1991-1998, 2005.

[26] J. P. David, K. Sabapathy, O. Hoffman, M. H. Idarraga, and E. F. Wagner, "JNK1 modulates osteoclastogenesis through both c-Jun phosphorylation-dependent and -independent mechanisms," Journal of Cell Science, vol. 115, no. 22, pp. 4317-4325, 2002.

[27] M. Cicek, A. Vrabel, C. Sturchio et al., "TGF- $\beta$ inducible early gene 1 regulates osteoclast differentiation and survival by mediating the NFATc1, AKT, and MEK/ERK signaling pathways," PLoS ONE, vol. 6, no. 3, Article ID e17522, 2011.

[28] J. Qiu, "Traditional medicine: a culture in the balance," Nature, vol. 448, no. 7150, pp. 126-128, 2007.

[29] J. Huang, L. Yuan, X. Wang, T. L. Zhang, and K. Wang, "Icaritin and its glycosides enhance osteoblastic, but suppress osteoclastic, differentiation and activity in vitro," Life Sciences, vol. 81, no. 10, pp. 832-840, 2007.

[30] T. P. Hsieh, S. Y. Sheu, J. S. Sun, M. H. Chen, and M. H. Liu, "Icariin isolated from Epimedium pubescens regulates osteoblasts anabolism through BMP-2, SMAD4, and Cbfa1 expression," Phytomedicine, vol. 17, no. 6, pp. 414-423, 2010.

[31] M. S. Wong, S. K. Mok, W. F. Chen et al., "Icariin protects against bone loss induced by oestrogen deficiency and activates oestrogen receptor-dependent osteoblastic functions in
UMR 106 cells," British Journal of Pharmacology, vol. 159, no. 4, pp. 939-949, 2010.

[32] J. Zhao, S. Ohba, M. Shinkai, U. I. Chung, and T. Nagamune, "Icariin induces osteogenic differentiation in vitro in a BMPand Runx2-dependent manner," Biochemical and Biophysical Research Communications, vol. 369, no. 2, pp. 444-448, 2008.

[33] T. P. Hsieh, S. Y. Sheu, J. S. Sun, and M. H. Chen, "Icariin inhibits osteoclast differentiation and bone resorption by suppression of MAPKs/NF- $\kappa \mathrm{B}$ regulated HIF- $1 \alpha$ and PGE2 synthesis," Phytomedicine, vol. 18, no. 2-3, pp. 176-185, 2011.

[34] C. Ma, J. Zhang, J. Fu, L. Cheng, G. Zhao, and Y. Gu, "Up-regulation of VEGF by MC3T3-E1 cells treated with curculigoside," Phytotherapy Research, vol. 25, no. 6, pp. 922 926, 2011.

[35] L. Jiao, D. P. Cao, L. P. Qin et al., "Antiosteoporotic activity of phenolic compounds from Curculigo orchioides," Phytomedicine, vol. 16, no. 9, pp. 874-881, 2009.

[36] Y. Wang, Y. Wang, L. Zhao et al., "Curculigoside isolated from Curculigo orchioides prevents hydrogen peroxide-induced dysfunction and oxidative damage in calvarial osteoblasts," Acta Biochimica et Biophysica Sinica, vol. 44, no. 5, pp. 431441, 2012.

[37] J. P. Hu, K. Nishishita, E. Sakai et al., "Berberine inhibits RANKL-induced osteoclast formation and survival through suppressing the NF- $\kappa \mathrm{B}$ and Akt pathways," European Journal of Pharmacology, vol. 580, no. 1-2, pp. 70-79, 2008.

[38] W. L. Hyun, H. S. Jung, H. N. Kim et al., "Berberine promotes osteoblast differentiation by Runx2 activation with p38 MAPK," Journal of Bone and Mineral Research, vol. 23, no. 8, pp. 1227-1237, 2008. 


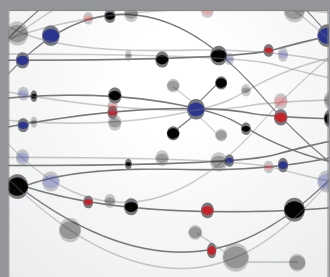

The Scientific World Journal
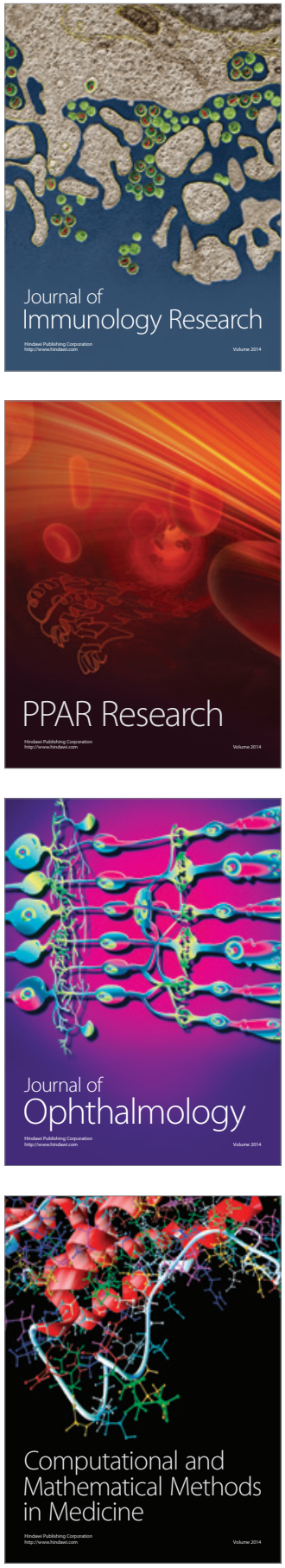

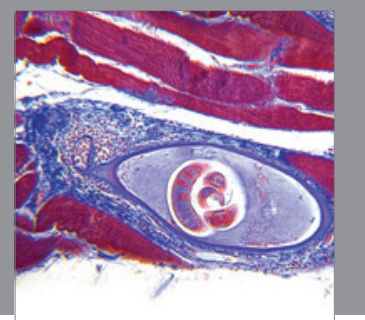

Gastroenterology

Research and Practice
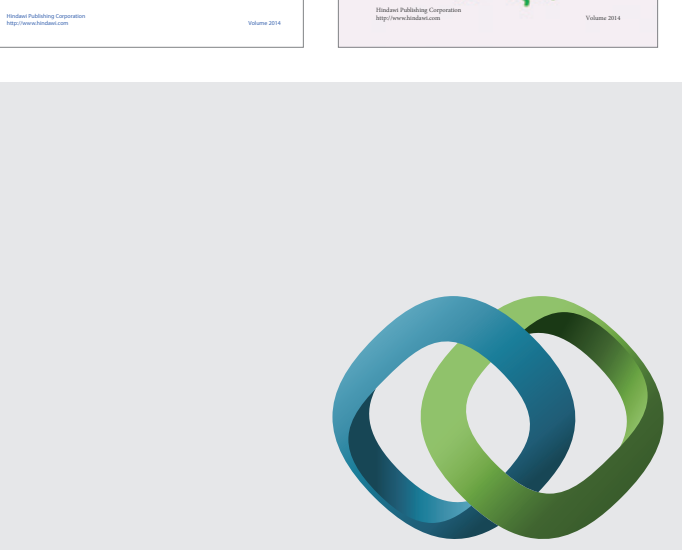

\section{Hindawi}

Submit your manuscripts at

http://www.hindawi.com
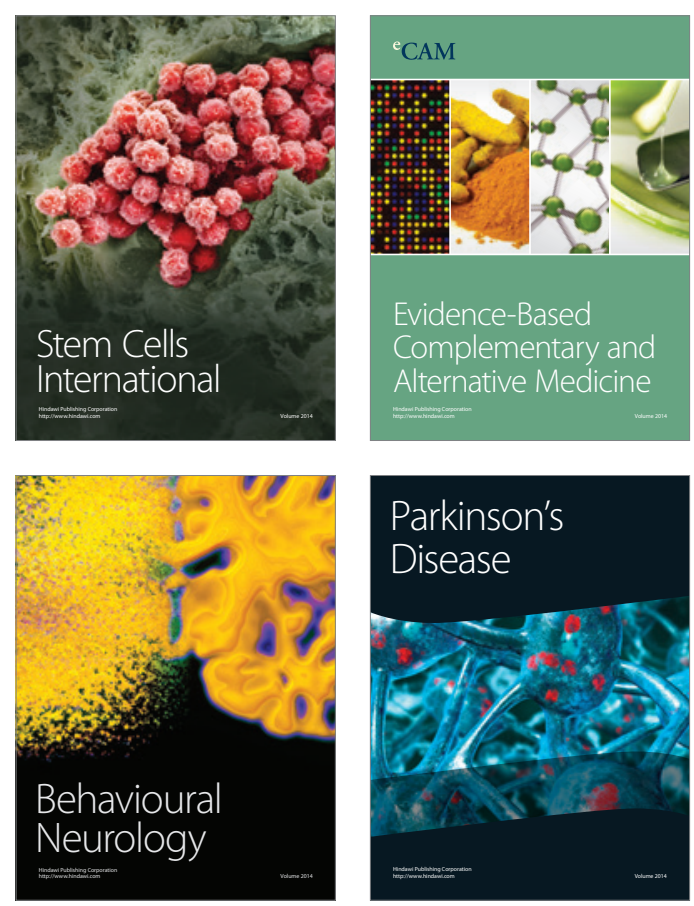

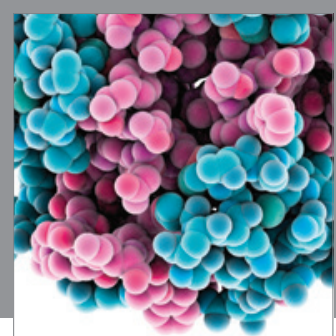

Journal of
Diabetes Research

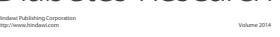

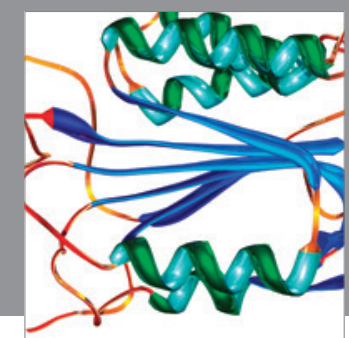

Disease Markers
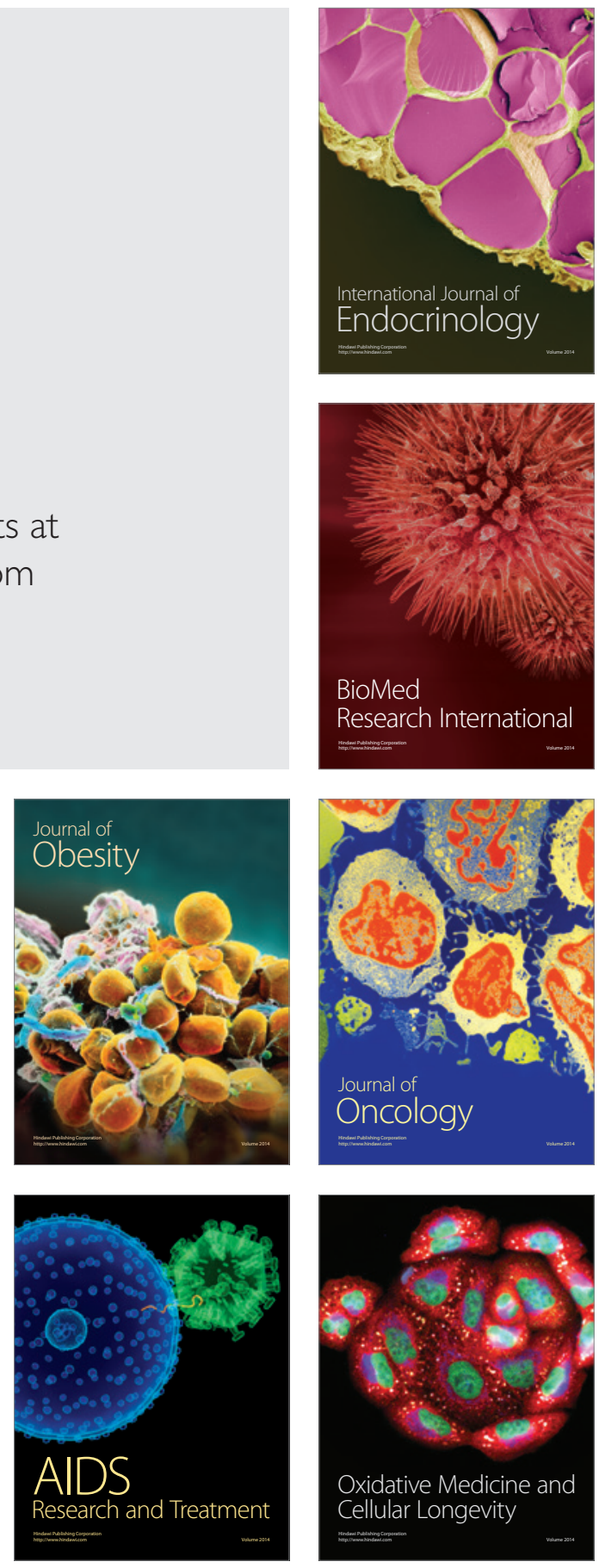